EPJ Web of Conferences 113,03001 (2016)

DOI: $10.1051 /$ epjconf/201611303001

(C) Owned by the authors, published by EDP Sciences, 2016

\title{
Weakly bound states with spin-isospin symmetry
}

\author{
A. Kievsky ${ }^{1, a}$ and M. Gattobigio ${ }^{2, b}$ \\ ${ }^{1}$ Istituto Nazionale di Fisica Nucleare, Largo Pontecorvo 3, 56127 Pisa, Italy \\ ${ }^{2}$ Université de Nice-Sophia Antipolis, Institut Non-Linéaire de Nice, CNRS, 1361 route des Lucioles, 06560 \\ Valbonne, France
}

\begin{abstract}
We discuss weakly bound states of a few-fermion system having spin-isospin symmetry. This corresponds to the nuclear physics case in which the singlet, $a_{0}$, and triplet, $a_{1}, n-p$ scattering lengths are large with respect to the range of the nuclear interaction. The ratio of the two is about $a_{0} / a_{1} \approx-4.31$. This value defines a plane in which $a_{0}$ and $a_{1}$ can be varied up to the unitary limit, $1 / a_{0}=0$ and $1 / a_{1}=0$, maintaining its ratio fixed. Using a spin dependant potential model we estimate the three-nucleon binding energy along that plane. This analysis can be considered an extension of the Efimov plot for three bosons to the case of three 1/2-spin-isospin fermions.
\end{abstract}

\section{Introduction}

In a series of papers $[1,2] \mathrm{V}$. Efimov has shown that three identical bosons interacting through a twobody short-range potential have a resonant spectrum at the unitary limit, $1 / a=0$, with $a$ the two-body scattering length. In this limit a geometrical series of bound states appears whose energies accumulate to zero. The ratio between the energies of two consecutive states is constant and, remarkably it results independent of the particular form of the interaction. This behavior has been denoted Efimov effect and its characteristic of being universal has given to this effect a particular relevance. In fact in the last two decades an enormous amount of work has been dedicated to study this effect in different fields as molecular, atomic, nuclear and particle physics.

The spectrum of the three-boson system close to the unitary limit is described by the Efimov equations (or Efimov radial law) that can be put in the following form

$$
\begin{gathered}
E_{3}^{n} /\left(\hbar^{2} / m a^{2}\right)=\tan ^{2} \xi \\
\kappa_{*} a=\mathrm{e}^{\left(n-n^{*}\right) \pi / s_{0}} \frac{\mathrm{e}^{-\Delta(\xi) / 2 s_{0}}}{\cos \xi},
\end{gathered}
$$

where $\Delta(\xi)$ is an universal function. A parametrization of this function in the range $-\pi<\xi<-\pi / 4$ can be found in Ref. [3]. The quantity $s_{0} \approx 1.00624$ is a universal number and $\kappa_{*}$ defines the energy $\hbar^{2} \kappa_{*}^{2} / m$ of the level $n=n^{*}$ at the unitary limit. Furthermore, knowing the value of $\kappa_{*}$ the complete spectrum is determined as a function of $a$. It should be noticed however that the above equations have been derived in the zero-range limit. In this limit the two-body energy results to be $E_{2}=\hbar^{2} / m a^{2}$ and,

\footnotetext{
a e-mail: kievsky@pi.infn.it

be-mail: mario.gattobigio@inln.cnrs.fr
} 
in addition, all the two-body low energy scattering observables can be written in terms of $a$, making this quantity a control parameter (see for instance Ref. [3] and references therein). When a two-body potential with finite range is considered, the Efimov radial law can be modified as it was discussed by the authors in Refs. [4, 5]. In the following we extend the discussion to the case in which the particles are 1/2-spin-isospin fermions [6].

\section{Efimov plot with 1/2-spin-isospin fermions}

The zero-range energy spectrum given by Eq.(1) is unbounded from below as has been shown first by Thomas [7]. Finite-range potentials cure this pathology modifying the energy spectrum. For values of $a$ much bigger than the range of the interaction Eq.(1) is still valid for the highest levels, however the ground and first excited levels show finite-range effects. In order to take into account these corrections the authors have proposed a modification to the Efimov radial low $[4,5]$,

$$
\begin{aligned}
E_{3}^{n} / E_{2} & =\tan ^{2} \xi \\
\kappa_{n}^{3} a_{B}+\Gamma_{n}^{3} & =\frac{\mathrm{e}^{-\Delta(\xi) / 2 s_{0}}}{\cos \xi} .
\end{aligned}
$$

Here there are two corrections, one coming from the two-body system and it can be taken into account by substituting $a$ with $a_{B}$, defined by $E_{2}=\hbar^{2} / m a_{B}^{2}$, with $E_{2}$ the two-body binding energy if $a>$ 0 , or the two-body virtual-state energy in the opposite case, $a<0$. The second correction is the introduction of a finite-range parameter, $\Gamma_{n}^{3}$, depending on the energy level, which produces a shift in the product $\kappa_{*} a_{B}$. Recently it has been shown that the value of the shift is almost the same for very different potentials in range and scale as the LM2M2 interaction of Aziz [8], a gaussian potential reproducing the values of $E_{2}$ and $a$ given by that interaction and a combination of yukawians, the MT-III interaction [9], describing the $s$-wave triplet $n-p$ state. Interestingly the value of the shift for the ground state in the three cases results to be almost the same, $\Gamma_{0}^{3} \approx 0.8[10]$.

In the following we would like to discuss the case of fermions having $1 / 2$ isospin symmetry in which the interaction is different in the singlet and triple state as for the $n-p$ system. In this case the experimental data are $a_{0}=-23.740 \pm 0.020 \mathrm{fm}$ and $a_{1}=5.424 \pm 0.003 \mathrm{fm}$. To describe these data we use a gaussian potential

$$
V(r)=V_{S} \mathrm{e}^{-r^{2} / r_{S}^{2}},
$$

with different strength in the singlet $(S=0)$ and triplet $(S=1)$ and having the same range (in the following we consider $r_{0}=r_{1}=1.65 \mathrm{fm}$ ). The values $V_{0}=-37.90 \mathrm{MeV}$ and $V_{1}=-60.575 \mathrm{MeV}$ approximate the experimental data and, for $S=1$, the deuteron binding energy is approximated too. Varying the strengths $V_{S}$ it is possible to explore the three-nucleon binding energy as the values of $a_{S}$ move toward the unitary limit. Among different possibilities we fix the ratio $a_{0} / a_{1}=-4.31$ to its experimental value and calculate the energy of the ground and first excited states. In Fig.1 we show the different planes (the three-body energy defines the third axis not shown in the figure) determined by the values of $a_{0}$ and $a_{1}$. When the two scattering lengths are equal, $a_{0} / a_{1}=1$, the corresponding plane is the boson plane for three equal particles very well studied (see for instance Ref. [4]). In the figure this is given by the (red) solid line. The (blue) solid line corresponds to what we call the nuclear plane since it contains the physical nuclear point, the continuation to the fourth quadrant given by the (blue) dashed line does not give new information since the exchange of the singlet and triplet potentials gives the same binding energy. The two axes correspond to planes in which one of the scattering lengths is on the unitary limit. The energy values of $E_{3}^{n}$ are different in the different planes however they collapse to the boson spectrum when the two scattering lengths are at the unitary limit. Using the gaussian potential given above the ground state energy in this limit results $E_{3}^{0} \approx 3.6 \mathrm{MeV}$. 




Figure 1. Different planes as function of the inverse of $a_{0}$ and $a_{1}$

The main results of the present study is given in Fig.2 where the binding momentum, defined as $\left(\hbar^{2} / m\right) K_{3}^{2}=E_{3}$, for the ground and excited states are shown as a function of $a_{1}$ (in units of $r_{*}$, the value of the effective range at the unitary limit) maintaining the ratio $a_{0} / a_{1}$ fixed around -4.31 . The three-fermion binding momenta are given by the (blue) solid line for the ground state and by the (blue) dot-dashed line for the first excited state. For comparison the binding momentum values in the case of three bosons are given too by the (black) solid and dashed lines. The (red) solid line is the two-body biding momentum. $K_{*}$ is the momentum at the unitary limit and both, the fermion and boson values, are the same. The excited state in this limit has a value of $K_{*} / 22.9$ showing a slight finite-range correction (the zero-range theory predicts the value $K_{*} / 22.7$ ). The (negative) values of the triplet scattering lengths, $a_{-}^{0}$ and $a_{-}^{1}$, at which the three-boson ground and excited states disappear into the three-nucleon continuum are shown in the figure. Their ratio of about 17.6 is well below the prediction of 22.7 given by the zero-range theory. More interesting is the fact that the excited state of the three-fermion system disappears into the $n-d$ continuum before matching the nuclear physical point (at $a_{*}^{1} \approx 20 \mathrm{fm}$ ). This is not the case for the three-boson system, the excited state remains bound and always below $E_{2}$. This fact has been studied before in the case of a system of three helium atoms. The present analysis shows that in the case of three nucleons the difference in the single and triple potential strengths is such that the excited state disappear into the $n-d$ continuum before reaching the physical point. Evidence of the presence of this state embedded in the $n-d$ continuum has been given in the literature $[11,12]$ and the curvature of the $n-d$ effective range function close to the threshold is one of this $[13,14]$. Finally, at the physical value, the two- and three-body energies are explicitly displayed. As it is well known, in order to reproduce the triton binding energy a three-body force has to be included.

The spectrum of the three-fermion system can be still described by Eq.(2) with a value of the shift depending on the ratio $a_{0} / a_{1}$. In the present analysis we found for the ground state level $\Gamma_{3}^{0} \approx-0.2$, a negative value. If we compare this value to the value of about 0.8 obtained in the case of three bosons we can conclude that there is a particular value of the ratio at which the shift $\Gamma_{3}^{0} \approx 0$. In this case Eq.(2) will be equivalent to the zero-range equation. However this would valid for the ground state level, the shift depends on the level and, as we have shown, the ratio with the excited state at the unitary limit is 22.9 (not 22.7) for a gaussian potential independently of the shift value. 




Figure 2. The binding momentum as a function of the inverse of $a_{1}$

\section{Conclusions and Outlook}

The present analysis focussed on the particularities of the Efimov plot in the case of 1/2-spin-isospin fermions. We have selected a particular way of constructing the Efimov plot in which the ratio of the singlet and triplet scattering lengths has been kept fixed. In this way the binding momentum and $a_{1}$ define a plane similar to the boson case, in particular the values at the unitary limit coincide. The present work has to be consider a first step in the study of the Efimov plot for three nucleons. At present the role of the three-body force and the predictions on systems with $A>3$ are underway.

\section{References}

[1] V. Efimov, Phys. Lett. B 33 (1970), 563

[2] V. Efimov, Sov. J. Nucl. Phys. 12 (1971), 589 [Yad. Fiz. 12, 10801090 (1970)]

[3] E. Braaten and H.-W. Hammer, Phys. Rep. 428 (2006), 259

[4] M. Gattobigio and A. Kievsky, Phys. Rev. A90, 012502 (2014)

[5] A. Kievsky, N.K. Timofeyuk and M. Gattobigio, Phys. Rev. A90, 032504 (2014)

[6] V. Efimov, Nuc. Phys. A 362 (1981), 45

[7] L.H. Thomas, Phys. Rev. 47, 903 (1935)

[8] R.A. Aziz and M.J. Slaman, J. Chem. Phys. 94, 8047 (1991)

[9] R.A. Malfliet and J.A. Tjon, Nuc. Phys. A 127, 161 (1969)

[10] A. Kievsky and M. Gattobigio, (2015) arXiv:1507.03402

[11] S.K. Adhikari, A.C. Fonseca and L. Tomio, Phys. Rev. C 26, 77 (1982)

[12] T. Frederico, I.D. Goldman and A. Delfino, Phys. Rev. C 37, 497 (1988)

[13] C.R. Chen, G.L. Payne, J.L. Friar and B.F. Gibson, Phys. Rev. C 39, 1261 (1989)

[14] A. Kievsky and M. Gattobiggio, Phys. Rev. A87, 052719 (2014) 